\title{
ATUAÇÃO FISIOTERAPÊUTICA NO TRAUMA RAQUIMEDULAR EM AMBIENTE HOSPITALAR ${ }^{1}$ PHYSICAL THERAPY PERFORMANCE IN SPINAL CORD INJURY IN A HOSPITAL ENVIRONMENT
}

\begin{abstract}
Lucas Lima Ferreira², Laís Helena Carvalho Marino ${ }^{3}$ e Simone Cavenaghi ${ }^{4}$
2 Fisioterapeuta graduado pela Fundação Educacional de Fernandópolis - FEF, São Paulo; especialista em Aprimoramento em Fisioterapia Hospitalar, pela Faculdade de Medicina de São José do Rio Preto - Famerp, São Paulo; mestrando em Fisioterapia pela Faculdade de Ciências e Tecnologia da Universidade Estadual Paulista Júlio de Mesquita Filho - FCT/ Unesp, campus de Presidente Prudente, São Paulo.

3 Fisioterapeuta graduada pela Universidade Paulista - Unip, São Paulo; doutoranda em Ciências da Saúde pela Faculdade de Medicina de Rio Preto - Famerp, São Paulo.

${ }^{4}$ Fisioterapeuta; doutora em Ciências da Saúde, pela Faculdade de Medicina de São José do Rio Preto - Famerp, São Paulo; coordenadora dos Cursos de Pós-Graduação Lato Sensu Fisioterapia na Saúde da Mulher, Terapia Manual e Postural e Fisioterapia em Geriatria; professora dos Cursos de Pós-Graduação Lato Sensu em Fisioterapia da Famerp.
\end{abstract}

Data de entrada do artigo: 21/05/2012 Data de aceite do artigo: 02/07/2012

\section{RESUMO}

Trauma raquimedular é uma lesão neurológica incapacitante que ocasiona paralisia, perda sensorial e disfunção fisiológica, envolvendo uma série de funções corporais com grande impacto na sociedade. Representa um problema de saúde pública e afeta principalmente a população de adultos jovens, do sexo masculino, podendo resultar em morte ou deficiência. Este estudo teve como objetivo agrupar e atualizar conhecimentos em relação à atuação fisioterapêutica no trauma raquimedular em ambiente hospitalar. Foi realizada uma pesquisa da literatura nas bases de dados SciELO, Lilacs e PubMed, cruzando-se os descritores spinal cord injury, cinesiotherapy, physiotherapy, mobilization, rehabilitation, physical therapy department, respiratory therapy e electrotherapy, no período de 2005 a 2010. A cinesioterapia é imprescindível desde a fase de choque medular, pois favorece a manutenção da amplitude de movimento articular e flexibilidade, além de prevenir complicações circulatórias decorrentes da imobilização prolongada no leito. A fisioterapia respiratória promove a higiene brônquica e a correção de padrões ventilatórios anormais e de algumas patologias respiratórias. A eletroterapia é um recurso ainda pouco utilizado por fisioterapeutas no âmbito hospitalar. O presente estudo evidenciou a escassez de pesquisas específicas que abordem a temática da atuação do fisioterapeuta sobre o trauma raquimedular em ambiente hospitalar.

Palavras-chave: traumatismos da medula espinhal; unidades hospitalares; serviço hospitalar de fisioterapia; modalidades de fisioterapia.

\section{ABSTRACT}

Spinal cord trauma is a disabling neurological injury, which causes paralysis, sensory loss and physiological dysfunction involving a number of bodily functions with great impact on society. Represents a public health problem that affects mainly the young adults, males, and may result in death or disability. This study aimed to collate and update knowledge in relation to physical therapy in spinal cord injury in a hospital environment. Literature search was conducted in the databases SciELO, PubMed and Lilacs crossing the spinal cord injury descriptors, kinesiotherapy, physiotherapy, mobilization, rehabilitation, physical therapy department, respiratory therapy and electrotherapy in the period 2005 to 2010. Kinesiotherapy is essential since the phase of spinal shock, since it favors the maintenance of joint range of motion and flexibility, and to prevent circulatory complications arising from prolonged immobilization in bed. Respiratory therapy promotes bronchial hygiene, correction of abnormal respiratory patterns and some respiratory diseases. The electrotherapy is a feature still little used by physiotherapists in the hospital. The present study showed the lack of specific studies that address the theme of the role of the physiotherapist on the spinal cord injury in a hospital environment.

Keywords: spinal cord injuries; hospital units; hospital physical therapy service; physical therapy modalities.

\footnotetext{
${ }^{1}$ Estudo realizado no Departamento de Fisioterapia do Hospital de Base Fundação Faculdade Regional de Medicina/
} Faculdade de Medicina de São José do Rio Preto - Funfarme/Famerp, sem fontes de financiamento. 


\section{I NTRODUÇÃO}

A lesão medular é uma das lesões mais devastadoras que uma pessoa pode sofrer, causando paralisia, perda sensorial e disfunção fisiológica, e envolvendo uma série de funções corporais ${ }^{(1)}$. O trauma raquimedular (TRM) é uma lesão neurológica incapacitante, com grande impacto na sociedade, representando um problema de saúde pública (2).

Afeta principalmente a população de adultos jovens, entre os 16 e 30 anos de idade, do sexo masculino, sendo a região cervical e a transição toracolombar os segmentos mais atingidos, e muitas vezes resulta em morte ou deficiência (3, 6). A incidência desse tipo de lesão é bastante variada nos diferentes países, e ainda não é bem definida no Brasil por não haver uma notificação eficaz dos casos e pela escassez de estudos epidemiológicos significativos ${ }^{(2)}$. Existem poucos dados sobre o TRM no Brasil, onde se estima uma incidência de dez mil novos casos por ano, principalmente devido ao trauma ${ }^{(4)}$.

Em muitos países, a lesão da medula espinhal ocorre em uma taxa anual de 20-40 indivíduos por milhão. A América do Norte vem apresentando uma incidência de 11 mil novos casos ao ano, a maioria devido a acidentes nas vias públicas e agressões físicas ${ }^{(3,5)}$. As principais causas de lesão medular no Brasil, levando-se em consideração as maiores cidades, são as lesões por armas de fogo, por meios violentos, e os acidentes de trânsito (2). Por outro lado, quedas, acidentes nas vias públicas e em mergulhos são vistos com certa frequência nos serviços de urgências (3).

Em relação à fisiopatologia do TRM, após a lesão mecânica primária, uma cascata de eventos é observada, o que leva à degeneração e morte do tecido neuronal. Dentre os componentes da lesão secundária, a isquemia/hipóxia é considerada um dos fatores mais importantes implicados na lesão do tecido neuronal (4). Na maioria dos casos de TRM, o mecanismo da lesão primária é a compressão aguda ou a laceração da medula espinal devido ao deslocamento ósseo ou hérnia de disco, após o deslocamento ou explosão da fratura ${ }^{(3)}$.

Nos primeiros minutos do TRM, são comuns complicações como a hipertensão arterial e disritmias cardíacas, seguidas da fase de choque, resultante da diminuição do tônus simpático e perda das respostas motora e sensitiva, com duração de três a seis semanas, caracterizada por manifestações cardiovasculares e neurológicas importantes, destacando-se a diminuição do retorno venoso pela vasodilatação periférica; a diminuição do volume sistólico e débito car- díaco; a bradicardia e a diminuição da tolerância ao exercício; a alteração da termorregulação; a diminuição do fluxo sanguíneo para os músculos em atividade e a paralisia flácida $(6,7)$.

A Associação Americana do Trauma Raquimedular (Asia - American Spine Injury Association) desenvolveu padrões para a avaliação e classificação neurológica do TRM, que apresentou grande aceitação em nível mundial, classificando a lesão medular em completa, quando as funções motoras e sensitivas encontram-se interrompidas abaixo do nível do trauma, e incompleta, quando existe função sensitiva e/ou motora preservada abaixo do nível da lesão ${ }^{(6,8)}$.

A fisioterapia é considerada como um componente importante na gestão dos pacientes em ambiente hospitalar por fornecer benefícios a curto e médio prazo ${ }^{(9)}$. Em nível hospitalar, o fisioterapeuta atua por meio de técnicas respiratórias, que incluem percussão, vibração, técnicas de tosse assistida, aspiração, dentre outras, que são eficazes na remoção de muco das vias aéreas ${ }^{(10)}$, e técnicas motoras como a cinesioterapia, por meio de exercícios passivos, ativoassistidos, ativos e resistidos (6).

Desta forma, o presente trabalho tem por objetivo agrupar e atualizar os conhecimentos em relação à atuação fisioterapêutica no trauma raquimedular em ambiente hospitalar.

\section{MÉTODOS}

Trata-se de uma atualização de literatura, na qual a busca foi realizada nas bases de dados eletrônicas Lilacs ${ }^{2}$, PubMed ${ }^{3}$ e SciELO ${ }^{4}$, no período de janeiro de 2005 a janeiro de 2010.

As palavras-chave usadas em várias combinações foram as seguintes: spinal cord injury, critical illness, cinesiotherapy, physical therapy, physiotherapy, exercises, training, active mobilization, mobilization, rehabilitation, physical therapy department, respiratory therapy e electrotherapy.

Os critérios de inclusão assim se caracterizaram: pesquisas nas línguas inglesa ou portuguesa, realizadas com seres humanos adultos, de 18 anos ou mais, de ambos os sexos, que abordassem a atuação da fisioterapia respiratória e/ ou motora no TRM em ambiente hospitalar, e que tinham sido publicados entre os anos de

\footnotetext{
2 Literatura Latino-Americana e do Caribe em Ciências da Saúde.

3 Banco de dados da National Library of Medicine.

${ }^{4}$ Scientific Electronic Library Online.
} 
2005 a 2010, bem como todos os tipos de desenho de estudo. Por outro lado, adotaram-se os critérios de exclusão a seguir: artigos que excluíram pacientes portadores de lesão medular de qualquer espécie; trabalhos com crianças ou adolescentes; pesquisas com lesados medulares fora do ambiente hospitalar; além de resumos de dissertações ou teses acadêmicas.

Foi realizada uma análise de títulos e resumos com vistas à obtenção de artigos potencialmente relevantes para a revisão. Pesquisa secundária foi realizada por meio da análise das referências bibliográficas dos estudos selecionados. Assim, foram triados 41 artigos sobre fisioterapia no trauma raquimedular, publicados no período selecionado, porém 30 destes foram excluídos por consistirem em estudos realizados em ambiente clínico ou laboratorial, restando apenas 11 artigos que preencheram os critérios de inclusão desse estudo.

\section{DESENVOLVI MENTO}

\subsection{Fisioterapia motora}

A fisioterapia motora por meio da cinesioterapia é efetiva na reabilitação do paciente lesado medular em todas as fases da lesão, pois proporciona maior independência funcional e melhora da qualidade de vida ${ }^{(6)}$. Dessa forma, Cavenaghi et al. (6), em um trabalho de atualização, verificaram a aplicabilidade e os efeitos da cinesioterapia na reabilitação de pacientes com trauma raquimedular durante a permanência hospitalar, e concluíram que há necessidade de novas pesquisas no campo da fisioterapia com foco na reabilitação funcional do paciente lesado raquimedular e na prevenção das comorbidades às quais eles estão expostos, principalmente durante a fase hospitalar.

Estudo realizado com nove indivíduos de ambos os sexos, portadores de TRM crônico em ambiente hospitalar, analisou as respostas para exercícios passivos e ativos por meio de um equipamento chamado Flexiciser (Flexiciser International Corp, Carlsbad, CA), dispositivo que poderia aumentar a resistência, o retorno venoso, o movimento e o tônus muscular, o controle de peso e dor, e, ao mesmo tempo, reduzir a espasticidade, o estresse, a depressão e o edema. Em duas sessões de 30 minutos de exercícios ativos de membros inferiores (MMII) e exercícios passivos de membros superiores (MMSS), e na análise dos parâmetros hemodinâmicos, pôdese verificar aumento significativo na frequência cardíaca (FC), pressão arterial sistólica (PAS) e consumo de oxigênio $\left(\mathrm{VO}_{2}\right)$ do repouso para o exercício. No entanto, a eficácia deste dispositivo para reabilitação em longo prazo com exercícios regulares para estes pacientes ainda é desconhecida ${ }^{(11)}$.

Chang et al. (12) avaliaram o uso de pranchas de inclinação como recurso da fisioterapia motora para pacientes em unidade de terapia intensiva (UTI) em toda a Austrália e verificaram que os fisioterapeutas nessas unidades são proativos na mobilização precoce do paciente crítico, obtendo, como resultados específicos do recurso utilizado, ganhos nas funções de facilitar a descarga de peso $(94,8 \%)$, prevenir contraturas musculares $(86 \%)$, melhorar a força dos MMII $(81 \%)$ e aumentar a excitação muscular $(70 \%)$. Essa técnica vem sendo mais utilizada em pacientes neurológicos $(63,8 \%)$ com longo prazo de permanência na UTI $(43,1 \%)$, associando ao tratamento de inclinação técnicas cinesioterapêuticas, como exercícios de MMSS $(93,1 \%)$ e exercícios respiratórios $(86,2 \%)$.

Um estudo de caso (13) descreveu o uso de tomografia computadorizada quantitativa (TCQp) para identificar as respostas musculoesqueléticas à aplicação de peso parcial em treinamento com esteira ergométrica na lesão medular incompleta, iniciado no ambiente hospitalar, com duração de sete meses de treinamento parcial. Varreduras de TCQp pré e pós-treinamento de MMII foram utilizadas para quantificar as mudanças no osso trabecular, no osso cortical e em tecidos moles. A densidade mineral óssea trabecular aumentou $5 \%$ (à direita) e $20 \%$ (à esquerda) na tíbia distal; as alterações na tíbia proximal e fêmur distal foram insignificantes. Houve aumentos na área de secção transversa do músculo, de $6 \%$ (à direita) e $12 \%$ (à esquerda) na parte inferior da perna, 7\% (à direita) e 5\% (à esquerda) na coxa. Os autores sugeriram que o treinamento em esteira pode levar a adaptações musculoesqueléticas positivas em locais clinicamente relevantes. Tais mudanças podem ser medidas em detalhe, utilizando-se a tomografia computadorizada quantitativa.

De Ruz et al. (14) avaliaram as mudanças que ocorrem na marcha de pacientes com lesão medular incompleta que foram tratados em nível hospitalar com um sistema de andar robótico em associação com a cinesioterapia convencional. Trata-se de um ensaio aberto, descritivo e prospectivo, envolvendo pacientes com lesões medulares níveis C2-L3 que foram classificados como graus C e D, de acordo com a escala Asia. Quarenta e cinco pacientes, com idade média de $44 \pm 14,3$ anos, completaram o estudo, sendo que $76 \%$ eram do sexo masculino e a lesão foi causada por um trauma em $58 \%$ dos casos. 
Aumentos estatisticamente significativos foram observados no número de sujeitos capazes de andar, na velocidade da caminhada, menor necessidade de ajuda técnica, na força nos MMII e na independência nas atividades da vida diária. O tratamento utilizando o sistema robótico em associação com a cinesioterapia convencional meIhora a capacidade de marcha em pacientes com lesão medular incompleta ${ }^{(14)}$.

\subsection{Fisioterapia respiratória}

A fisioterapia respiratória pode ser utilizada em pacientes críticos com o objetivo de prevenir e/ou tratar complicações respiratórias. Para isso, geralmente é usada uma combinação de procedimentos que objetivam a reexpansão e a remoção de secreções nas vias aéreas ${ }^{(15)}$.

Mueller et al. (16) objetivaram determinar o nível de ventilação que lesados medulares, com paraplegia e tetraplegia, podiam sustentar por 10 a 20 minutos, como base para orientar o treinamento de resistência dos músculos respiratórios nesses pacientes. Trata-se de um estudo-piloto com dois grupos de 14 pacientes: oito pacientes com paraplegia e seis com tetraplegia, nos quais foram realizados testes de resistência muscular respiratória em três diferentes intensidades, a $20 \%$, a $40 \%$ e a $60 \%$ da ventilação voluntária máxima (VVM). A resistência respiratória foi analisada separadamente para pacientes com paraplegia e tetraplegia. Os resultados demonstraram que os tempos de resistência respiratória média foram de 46,0, 18,9 e 4,2 minutos em $20 \%, 40 \%$ e $60 \%$ da VVM, em pacientes com tetraplegia, e 51,8, 38,8 e 12,2 minutos em pacientes com paraplegia. A duração diferiu significativamente em $60 \%$ da VVM entre os grupos. Os referidos autores concluíram que a ventilação por minuto, para realizar o treinamento de resistência dos músculos respiratórios, pode ser fixada em cerca de $40 \%$ em pacientes com tetraplegia e cerca de $60 \%$ da VVM em pacientes com paraplegia, pois estes níveis podem ser sustentados por 10 a 20 minutos ${ }^{(16)}$.

Shavelle et al. (17), realizaram uma análise de regressão logística em um conjunto de dados de 1.986 pessoas-ano para verificar a sobrevivência de pacientes com lesão medular traumática, que são dependentes do ventilador e de reabilitação na alta hospitalar, pelo menos um ano após a lesão. Nos dados analisados, ocorreram 319 pessoas feridas, entre 1973 e 2003. Como resultados, verificou-se que os principais fatores relacionados à sobrevivência a longo prazo foram os seguintes: idade, tempo de lesão, nível neurológico e grau de complexidade da lesão. A expec- tativa de vida era menor do que estimativas anteriores. Pneumonia e outras afecções respiratórias foram as principais causas de morte, mas representaram apenas $31 \%$ dos óbitos de causas conhecidas (17).

Uma revisão sistemática ${ }^{(18)}$ com estudos de avaliação de treinamento físico (TF) e treinamento muscular inspiratório (TMI) para a meIhoria da função respiratória em pacientes com TRM identificou 13 estudos (cinco TF e oito TMI). Os artigos foram marcados pela sua qualidade metodológica, utilizando-se a pontuação do Banco de Dados da Fisioterapia por Evidências (PEDro). Os resultados apontaram que a comparação do estudo foi comprometida por diversos desenhos de pesquisa, amostras de pequenas dimensões, heterogeneidade nas populações, protocolos e medidas de resultados estudadas. Com base na literatura atual, não há provas de nível II que confiabilizem o TF como uma intervenção para melhorar a força e a resistência respiratória e provas de nível IV para apoiar o TMI como uma intervenção que poderia melhorar, em repouso e no exercício, a função respiratória em lesados medulares. Também não há provas de nível IV para apoiar o TMI como uma intervenção que poderia diminuir a dispneia e melhorar a função respiratória em pessoas com TRM. Em suma, não existem dados suficientes para apoiar fortemente a utilização do TF ou TMI para melhorar a função respiratória em pessoas com TRM. Há alguma evidência de eficácia de ambas as técnicas; no entanto, tal evidência não é da melhor qualidade possível ${ }^{(18)}$.

\subsection{Recursos eletroterapêuticos}

Os recursos elétricos com finalidade terapêutica ainda são pouco utilizados por fisioterapeutas no nível hospitalar, especialmente no que concerne ao tratamento do paciente lesado raquimedular, tendo seus benefícios, bem como seus riscos, não claramente definidos (19). Com base nessa afirmação, Lee et al. (19) realizaram um estudo de caso clínico que avaliou as modalidades de estimulação não invasivas para aumentar a tosse e ajudar a decanulação da traqueostomia em um paciente com tetraplegia por lesão medular. Um homem de 65 anos com tetraplegia (nível C4 - Asia C) atrasou a reabilitação devido a uma traqueostomia e pneumonia recorrente, resultantes principalmente de tosse ineficaz. A estimulação elétrica de superfície (EES) da musculatura abdominal anterior foi aplicada para obter uma tosse eficaz e permitir a decanulação. Os resultados demonstraram que, no início, a adição da EES causou aumento da pressão expiratória máxima $(80 \%)$, tosse com 
pressão expiratória máxima (67\%) e taxa de pico de fluxo expiratório (11\%). Três semanas após o treino começar na sequência de um programa de EES com tosse assistida e voluntária, o paciente foi decanulado e, após testar um ano mais tarde, a EES com eletrodos colocados posterolaterais também produziu um aumento das tentativas de tosse voluntária. Os autores citados concluíram que a EES não invasiva pode potencialmente ajudar na decanulação da traqueostomia ${ }^{(19)}$.

A ossificação heterotópica é uma complicação frequente após a lesão medular (20). Assim, um estudo avaliou a resposta da ossificação heterotópica das articulações coxofemorais à estimulação elétrica neuromuscular (EENM) em seis pacientes tetraplégicos após TRM, por um período médio de 16,6 meses, sendo avaliados radiologicamente. Foi identificada a melhora radiológica em dois pacientes e a não progressão do quadro nos demais. A EENM em lesados medulares pode ser um método válido no tratamento da ossificação heterotópica e na prevenção de sua progressão ${ }^{(20)}$.

Um estudo (21) realizado com o objetivo de investigar as variáveis cardiorrespiratórias (pressão arterial, frequência cardíaca, consumo de oxigênio, produção de dióxido de carbono e ventilação minuto) durante a EENM do quadríceps em portadores de lesão medular, com a participação de dez pacientes (cinco paraplégicos e cinco tetraplégicos), por meio de um protocolo que consistiu em dez minutos de repouso, 20 minutos de EENM e dez minutos de recuperação, obteve como resultados baixos valores de con- sumo de oxigênio e produção de dióxido de carbono. Os paraplégicos apresentaram rápida cinética dos gases e os tetraplégicos, lenta cinética dos gases. Houve o aumento da PAS e da frequência cardíaca. Os valores das variáveis cardiorrespiratórias foram inversamente proporcionais ao nível de lesão, ou seja, quanto maior o nível de lesão, menores os valores. Portanto, a maioria dos pacientes apresentou algumas limitações nas respostas cardiorrespiratórias, indicando realização de exercício exaustivo, mas apresentaram capacidade de realização de exercício induzido artificialmente, possivelmente devido aos benefícios da EENM (21).

\section{CONSI DERAÇÕES FI NAIS}

A fisioterapia precoce em âmbito hospitalar encontra-se focada na reabilitação motora por meio da cinesioterapia, na intervenção respiratória por intermédio da higiene brônquica e treinamento dos músculos respiratórios e na utilização de recursos como a eletroterapia a fim de estimular musculaturas atrofiadas por desnervação, atuando com o objetivo primário de prevenir maiores complicações, proporcionar maior independência funcional e melhorar a qualidade de vida dos pacientes atingidos. Ressalta-se a escassez de estudos específicos que abordem a temática da atuação do fisioterapeuta sobre o TRM no ambiente hospitalar. Dessa forma, sugerese que novos estudos sejam realizados na área da fisioterapia, enfocando os aspectos específicos da reabilitação do lesado medular desde a sua admissão até a alta hospitalar.

\section{REFERÊNCI AS}

(1) Pellatt GC. Patients, doctors, and therapists perceptions of professional roles in spinal cord injury rehabilitation: Do they agree? J Interprof Care. 2007 Mar; 21(2): 165-77.

(2) Custódio NRO, Carneiro MR, Feres CC, Lima GHS, Jubé MRR, Watanabe LE et al. Lesão medular no Centro de Reabilitação e Readaptação Dr. Henrique Santillo (CRER - GO). Coluna/ Columna. 2009; 8(3):265-8.

(3) Leal-Filho MB, Borges G, Almeida BR, Aguiar AAX, Vieira MACS, Dantas KS et al. Spinal cord injury: epidemiologycal study of 386 cases with emphasis on those patients admitted more than four hours after the trauma. Arq Neuro-Psiquiatr. 2008 jun; 66(2-B):365-8.
(4) Falavigna A, Teles AR, Velho MC, Kleber FD. Effects of hyperbaric oxygen therapy after spinal cord injury: systematic review. Coluna/ Columna. 2009; 8(3):330-6.

(5) Carlson KF, Wilt TJ, Taylor BC, Goldish GD, Niewoehner CB, Shamliyan TA et al. Effects of exercise on disorders of carbohydrate and lipid metabolism in adults with traumatic spinal cord injury: systematic review of the evidence. J Spinal Cord Med. 2009; 32(4):361-78.

(6) Cavenaghi S, Gama D, Valério NI, Marino LHC, Ramirez C. Aplicabilidade intra-hospitalar da cinesioterapia no trauma raquimedular. Arq Ciênc Saúde. 2005 out/dez; 12(4):213-5. 


\section{REFERÊNCI AS}

(7) Sidorov EV, Townson AF, Dvorak MF, Kwon BK, Steeves J, Krassioukov A. Orthostatic hypotension in the first month following acute spinal cord injury. Spinal Cord. 2008 Jan; 46(1): 65-9.

(8) Masri(y) WS El. Traumatic spinal cord injury: the relationship between pathology and clinical implications. Trauma. 2006 Jan; 8(1) : 2946.

(9) Zeppos L, Patman S, Berney S, Adsett JA, Bridson JM, Paratz JD. Physiotherapy intervention in intensive care is safe: an observational study. Aust J Physiother. 2007; 53(4):279-83.

(10) Walker J. Spinal cord injuries: acute care management and rehabilitation. Nurs Stand. 2009 Jun; 23(42): 47-56.

(11) Astorino TA, Tyerman N, Wong K, Harness E. Efficacy of a new rehabilitative device for individuals with spinal cord injury. J Spinal Cord Med. 2008; 31(5):586-91.

(12) Chang AT, Boots R, Hodges PW, Paratz J. Standing with assistance of a tilt table in intensive care: a survey of Australian physiotherapy practice. Aust J Physiother. 2005; 50(1):51-4.

(13) Coupaud S, Jack LP, Hunt KJ, Allan DB. Muscle and bone adaptations after treadmill training in incomplete spinal cord injury: a case study using peripheral quantitative computed tomography. J Musculoskelet Neuronal I nteract. 2009 Oct/Dec; 9(4):288-97.

(14) De Ruz EA, Alcobendas-Maestro M, Casado-López R, Muñoz-Gonzalez A, FloridoSánchez MA, González-Valdizán E. A robotic system for gait re-education in patients with an incomplete spinal cord injury. Rev Neurol. 2009 Dec; 49(12):617-22.

(15) Jerre G, Beraldo MA, Silva TJ, Gastaldi $A$, Kondo $C$, Leme $F$ et al. Fisioterapia no paciente sob ventilação mecânica. Rev Bras Ter Intensiva. 2007 jul/set; 19(3): 399-407.

(16) Mueller G, Perret C, Spengler CM. Optimal Intensity for respiratory muscle endurance training in patients with spinal cord injury. J Rehabil Med. 2006 Nov; 38(6):381-6.

(17) Shavelle RM, DeVivo MJ, Strauss DJ, Paculdo DR, Lammertse DP, Day SM. Long-term survival of persons ventilator dependent after spinal cord injury. J Spinal Cord Med. 2006; 29(5):511-9.

(18) Sheel AW, Reid WD, Townson AF, Ayas NT, Konnyu KJ. Effects of exercise training and inspiratory muscle training in spinal cord injury: a systematic review. J Spinal Cord Med. 2008; 31(5): 500-8.

(19) Lee BB, Boswell-Ruys C, Butler JE, Gandevia SC. Surface functional electrical stimulation of the abdominal muscles to enhance cough and assist tracheostomy decannulation after high-level spinal cord injury. J Spinal Cord Med. 2008; 31(1):78-82.

(20) Ferreira DBJ, Lippelt HC, Cliquet-Júnior A. Estimulação elétrica neuromuscular na reversão da ossificação heterotrópica. Acta Ortop Bras. 2006; 14(2):72-4.

(21) Paolillo FR, Paolillo AR, Cliquet-Júnior A. Respostas cardiorrespiratórias em pacientes com traumatismo raquimedular. Acta Ortop Bras. 2005; 13(3): 149-52.

\footnotetext{
Endereços para correspondência:

Lucas Lima Ferreira

lucas_lim21@hotmail.com

Laís Helena Carvalho Marino

laiscmarino@gmail.com

Simone Cavenaghi

sicavenaghi.@ig.com.br
} 